\title{
Article \\ Anti-Inflammatory Activity of a Demineralized Bone Matrix: An In Vitro Pilot Study
}

\author{
Layla Panahipour ${ }^{1}$ (), Anes Omerbasic ${ }^{1}$, Azarakhsh Oladzad Abbasabadi ${ }^{1}$, Jila Nasirzade ${ }^{1}$, Zahra Kargarpour $^{1}$ \\ and Reinhard Gruber 1,2,3,*
}

1 Department of Oral Biology, Medical University of Vienna, 1090 Vienna, Austria; layla.panahipour@meduniwien.ac.at (L.P.); anes.omerbasic@meduniwien.ac.at (A.O.); azarakhsh.oladzadabbasbadi@meduniwien.ac.at (A.O.A.); jila.nasirzaderajiri@meduniwien.ac.at (J.N.); zahra.kargarpooresfahani@meduniwien.ac.at (Z.K.)

2 Department of Periodontology, School of Dental Medicine, University of Bern, 3010 Bern, Switzerland

3 Austrian Cluster for Tissue Regeneration, 1200 Vienna, Austria

* Correspondence: reinhard.gruber@meduniwien.ac.at

check for

updates

Citation: Panahipour, L.; Omerbasic,

A.; Oladzad Abbasabadi, A.;

Nasirzade, J.; Kargarpour, Z.; Gruber,

R. Anti-Inflammatory Activity of a

Demineralized Bone Matrix: An In

Vitro Pilot Study. Appl. Sci. 2022, 12,

876. https://doi.org/10.3390/

app12020876

Academic Editors: Antonio Scarano and Yin Xiao

Received: 22 October 2021

Accepted: 12 January 2022

Published: 15 January 2022

Publisher's Note: MDPI stays neutral with regard to jurisdictional claims in published maps and institutional affiliations.

Copyright: (c) 2022 by the authors. Licensee MDPI, Basel, Switzerland. This article is an open access article distributed under the terms and conditions of the Creative Commons Attribution (CC BY) license (https:// creativecommons.org/licenses/by/ $4.0 /)$.

\begin{abstract}
Demineralized bone matrix (DBM) is commonly used for the reconstruction of bone defects. Early graft consolidation involves a transient inflammatory process. It is, however, unclear whether DBM can modulate this process. To test this possibility, we prepared acid lysates of demineralized ground cortical (DGC) and moldable demineralized fibers (MDF). Murine RAW 264.7 and primary bone marrow macrophages were exposed to acid lysates of DGC and MFD prior to provoking an inflammatory response with lipopolysaccharide (LPS). Similarly, murine ST2 mesenchymal cells were exposed to DGC and MFD with and without interleukin $1 \beta$ (IL1) and TNF $\alpha$. We show here that acid lysates of DGC and MFD reduced the expression of IL1 and IL6 in RAW 264.7 macrophages, as determined by RT-PCR and, for IL6, by immunoassay. This response was confirmed with primary macrophages. Likewise, desalted acid lysates exert anti-inflammatory properties on RAW 264.7 cells and in ST2 cells, the forced expression of IL6, inducible nitric oxide synthase (iNOS) and chemokine ligand 5 (CCL5) was reduced. These in vitro findings suggest that DGC and MFD lower the inflammation-induced expression of inflammatory mediators in murine cell-based bioassays.
\end{abstract}

Keywords: demineralized bone matrix; inflammation; moldable demineralized fibers; demineralized ground cortical; allografts; bone regeneration; macrophages; resolution

\section{Introduction}

Demineralized bone matrix (DBM) is used to reconstruct bony defects [1], including in dental [2,3], trauma [4] and spinal surgery [5]. DBM provides an osteophilic surface where new bone can be laid, thereby allowing the ingrowth of new bone into the defect site, ideally bridging the defect [6]. The conglomerate of the DBM, the new bone, and the concomitant bone marrow undergoes remodeling and creeping substitution of the DBM, thus allowing a restitutio ad integrum of the bone and a reshaping of the anatomical contours of the former defect site [6]. Demineralized bone is derived from cadaver bone that undergoes multiple steps of processing until it is available as a ready-to-use allograft [1]. This processing pays particular attention to maintain the activity of the growth factors stored in the extracellular matrix, most of all the members of the bone morphogenetic protein (BMP) family with their unique osteoinductive properties $[7,8]$. DBM is thus equipped with the ability to initiate de novo bone formation at ectopic sites, which is usually tested in rodent models $[9,10]$. Hence, there is evidence that DBM allografts are a source of growth factors that can potentially support bone regeneration, resulting in graft consolidation in a patient.

Bone is a rich source of TGF- $\beta$ [11,12]. We have recently identified TGF- $\beta$ to be released into a liquid fraction from bone allografts, e.g., demineralized ground cortical (DGC) and 
moldable demineralized fibers (MDF) [13]. Apart from the growth factor activity, TGF- $\beta$ is thought to have anti-inflammatory functions [14]. It is therefore reasonable to assume that DGC and MDF exert an anti-inflammatory activity. Surprisingly, however, allografts are usually not tested for their potential anti-inflammatory activity [1]. It would be interesting to know if allografts exert such activity. This is because a transient local inflammation occurs during early bone regeneration [15] and, if not resolved, the chronic inflammation causes the pathological event of inflammatory osteolysis [16]. Theoretically, upon creeping substitution, DBM could potentially exert inflammation-modulating activity.

To determine the possible anti-inflammatory activity of DGC and MDF, we took the advantage of our previously established protocol to prepare acid lysates of bone [12] and dentin [17]. We then applied the lysates of DGC and MDF to our established in vitro inflammation assay. Our bioassays include murine RAW 264.7 macrophages and primary bone marrow-derived macrophages being exposed to bacterial endotoxins [18], as well as murine ST2 bone marrow-derived mesenchymal cells [19] and human gingival fibroblasts being exposed to inflammatory cytokines interleukin $1 \beta$ and TNF $\alpha$ [20]. The modulation of the inflammatory response is measured by changes in gene expression, including IL6, and by the intensity and nuclear translocation of phosphorylated p65 [21]. IL1 as well as IL6 are lead cytokines that represent a large panel of inflammatory mediators, all of which initiate and enhance an inflammatory process that is resolved in a physiologic situation of wound healing [22] and fracture repair [15] — but, if not being resolved, becomes the main cause of tissue destruction [23]. Inducible nitric oxide synthases (iNOS) is also involved in fracture healing [24] and mesenchymal cells expressing chemokine ligand 5 (CCL5; also known as RANTES) support revascularization [25]. In this pilot study, we show in vitro data suggesting that acid lysates prepared from DGC and MDF lower the expression of inflammatory mediators.

\section{Material and Methods}

\subsection{Preparation of Acid Lysates of DGC and MDF}

Demineralized ground cortical (DGC, AlloGraft DGC, Straumann, Basel, Switzerland; $1.2 \mathrm{~cm}^{3}$, around $0.7 \mathrm{~g}$; lot IDs: 2010131-3098, 2010131-3070, 2010131-3102, 20101313105, 2010131-3087, 2010131-3067, 2010131-3083) and moldable demineralized fibers (MDF, OraGRAFT ${ }^{\circledR}$ Prime, LifeNet Health Europe $\mathrm{GmbH}$, Vienna, Austria, $1.0 \mathrm{~cm}^{3}$, around $0.4 \mathrm{~g}$; IDs: 1814212-3196, 1814212-3144, 1814212-3165, 1814212-3193, 1814212-3158, 1814212-3178, 1814212-3134) were submerged to reach $0.1 \mathrm{~g} / \mathrm{mL}$ in $1 \mathrm{M} \mathrm{HCl}$ and left shaking overnight at room temperature. Acid lysates from DGC and MDF were collected through one centrifugation step at $20,000 \times g$ for five minutes. The $\mathrm{pH}$ was then neutralized with $1.0 \mathrm{M}$ $\mathrm{NaOH}$ and filtered sterile $(0.2 \mu \mathrm{m}, \mathrm{VWR}$ international, Radnor, PA, USA). After processing, we obtained around $10 \mathrm{~mL}$ and $6 \mathrm{~mL}$ of DGC and MDF lysates, respectively. The lysates were stored in aliquots at $-20^{\circ} \mathrm{C}$. The lysates were prepared individually from each lot and experiments represent individual lots. Lysates from lots were only pooled when not reaching enough volume for a given experiment. For desalting, acid lysates were prepared with $1.0 \mathrm{M} \mathrm{HCl}$ as indicated but the $\mathrm{pH}$ was neutralized with $10.0 \mathrm{M} \mathrm{NaOH}$ to keep the dilution of the sample low. Then, the lysates were subjected to a PD SpinTrap G-25, a microspin column that is designed for desalting and buffer exchange of biological sample following the instructions of the manufacturer (Sigma Aldrich, St. Louis, MO, USA). The new buffer was the serum-free medium.

\subsection{Murine RAW 264.7 and Bone Marrow-Derived Macrophages}

RAW 264.7 macrophage cells (LGC Standards, Wesel, Germany) were expanded at $3 \times 10^{5}$ cells $/ \mathrm{cm}^{2}$ into 24 -well plates in growth Dulbecco's Modified Eagle Medium (DMEM, Sigma Aldrich), 10\% fetal calf serum (Bio\&Sell GmbH, Nuremberg, Germany) and antibiotics (Sigma Aldrich). Bone marrow cells were collected from the femurs and tibias of female Balb/c mice aged 6-8 weeks. Bone marrow cells were seeded at $1 \times 10^{6} \mathrm{cells} / \mathrm{cm}^{2}$ into 24-well plates and grown for 7 days in $\alpha$ MEM (Gibco, New York, NY, USA) sup- 
plemented with $10 \%$ fetal bovine serum (Invitrogen, Grand Island, NY, USA), antibiotics (Sigma Aldrich) and $30 \mathrm{ng} / \mathrm{mL}$ M-CSF (Prospec, Ness-Ziona, Israel). Cells were preexposed to $25 \%$ acid lysates from DGC and MDF for $30 \mathrm{~min}$ before being exposed to $100 \mathrm{ng} / \mathrm{mL}$ LPS (Escherichia coli 0111: B41; Sigma Aldrich) for $24 \mathrm{~h}$ [18]. To test for the involvement of TGF- $\beta$ signaling, the TGF- $\beta$ receptor I kinase inhibitor SB431542 (Calbiochem, Merck, Billerica, MA, USA) was added at $10 \mu \mathrm{M}$ [13]. In another set of experiments, RAW 264.7 cells were exposed to $10-300 \mathrm{mM} \mathrm{NaCl}$ prior to the LPS challenge. Then, RT-PCR and immunoassays were performed.

\subsection{ST2 Mesenchymal Stromal Cell Line and Human Gingival Fibroblasts}

The ST2 mesenchymal stromal cell line was originally isolated from mouse bone marrow (RIKEN Cell Bank, Tsukuba, Japan). Tissue samples of human gingiva were harvested from the extracted third molars of three young and healthy patients who had given informed and written consent (Ethics Committee of the Medical University of Vienna; EK NR 631/2007). ST2 cells and gingival fibroblasts from pooled donors were plated in growth medium consisting of DMEM, $10 \%$ fetal calf serum (Bio\&Sell $\mathrm{GmbH}$ ) and antibiotics (Sigma Aldrich) at 30,000 cells $/ \mathrm{cm}^{2}$ into culture dishes. Cells were pre-exposed to $25 \%$ acid lysates from DGC and MDF for 30 min before being stimulated with IL1 $\beta$ and TNF $\alpha$ (ProSpec-Tany TechnoGene Ltd., Rehovot, Israel), both at $10 \mathrm{ng} / \mathrm{mL}$, in the serum-free medium for $24 \mathrm{~h}$ [20].

\subsection{RT-PCR and Immunoassay}

Total RNA was isolated with the ExtractMe total RNA kit (Blirt S.A., Gdańsk, Poland). Reverse transcription (RT) was performed with the LabQ FirstStrand cDNA Synthesis Kit (LabQ, Labconsulting, Vienna, Austria). Reverse transcription-polymerase chain reaction (RT-PCR) was conducted (LabQ, Labconsulting, Vienna, Austria) on a CFX Connect ${ }^{\mathrm{TM}}$ Real-Time PCR Detection System (Bio-Rad Laboratories, Hercules, CA, USA). Primer sequences were mIL6_F GCT ACC AAA CTG GAT ATA ATC AGG A; mIL6_R CCA GGT AGC TAT GGT ACT CCA GAA; mIL1ß_F AAG GGC TGC TTC CAA ACC TTT GAC; mIL1 $\beta$ _R ATA CTG CCT GCC TGA AGC TCT TGT; miNOS_F GGTGAAGGGACTGAGCTGTT; miNOS_R ACGTTCTCCGTTCTCTTGCAG; mCCL5_F CCTGCTGCTTTGCCTACCTC; mCCL5_R ACACACTTGGCGGTTCCTTC; mGAPDH_F AAC TTT GGC ATT GTC GAA CG; mGAPDH_R GGA TGC AGG GAT GAT GTT CT; hIL6_F GAA AGG AGA CAT GTA ACA AGA GT; hIL6_R GAT TTT CAC CAG GCA AGT CT; hIL8_F AAC TTC TCC ACA ACC CTC TG; hIL8_R TTGGCAGC CTTCCTGATTTC; hGAPDH_F AAG CCA CAT CGC TC; hGAPDH_R AGA CAC GCC CAA TAC GAC CAA ATC C. For primers on proliferation markers, see Supplementary Table S1. The mRNA levels were calculated by normalizing to the housekeeping gene GAPDH using the $\Delta \Delta \mathrm{Ct}$ method. The immunoassay was performed with the mouse IL6 and human IL8 Quantikine ELISA kit (R\&D Systems, Minneapolis, MN, USA).

\subsection{Immunofluorescence}

RAW 264.7 macrophages were plated in growth medium onto Millicell ${ }^{\circledR}$ EZ slides (Merck KGaA, Darmstadt, Germany). The following day, cells were treated with serum-free medium overnight. The next day, cells were exposed to 25\% DGC and MDF followed by $100 \mathrm{ng} / \mathrm{mL}$ LPS for $10 \mathrm{~min}$ each. Cells were then fixed in paraformaldehyde and blocked in 5\% bovine serum albumin (BSA) and 0.3\% Triton X-100 in phosphate-buffered saline (PBS) at room temperature, after which permeabilization with $0.1 \%$ Triton $\mathrm{X}-100$ took place. The cells were then incubated with rabbit anti-human NFkB p65 (\#8242; Cell Signaling Technology, Cambridge, UK) at $4{ }^{\circ} \mathrm{C}$ overnight. Detection was performed with the goat antirabbit Alexa 488 secondary antibody (CS-4412, Cell Signaling Technology). Finally, cells were washed and mounted onto glass slides. Images were captured under a fluorescent microscope (Axio Imager M2, Carl Zeiss AG, Oberkochen, Germany). 


\subsection{Statistical Analysis}

Statistical analysis comparing the inflammation group alone versus the DGC and MDF groups was based on a Friedmann test using GraphPad Software (Version 8, San Diego, CA, USA). To compare the DGC and MDF groups, a Wilcoxon matched-pairs signed rank test was performed that was in neither case significant and, thus, the $p$-values are not shown.

\section{Results}

\subsection{Acid Lysates of DGC and MDF Do Not Affect the Viability of RAW 264.7 Macrophages}

We first determined whether or not DGC and MDF can change the viability of the RAW 264.7 cells. The RAW 264.7 cells were grown in the presence of 12,25 and $50 \%$ allograft lysates for $24 \mathrm{~h}$ followed by a classical MTT viability assay. As indicated in Supplementary Figure S1, there was no considerable change in cell viability. Consistently, Figure 1 shows that RAW 264.7 cells exposed to LPS undergo the expected morphological changes typical for dendritic cells [26]. No detachment or other obvious signs of cell death occurred in the presence of $25 \%$ desalted DGC and MDF.
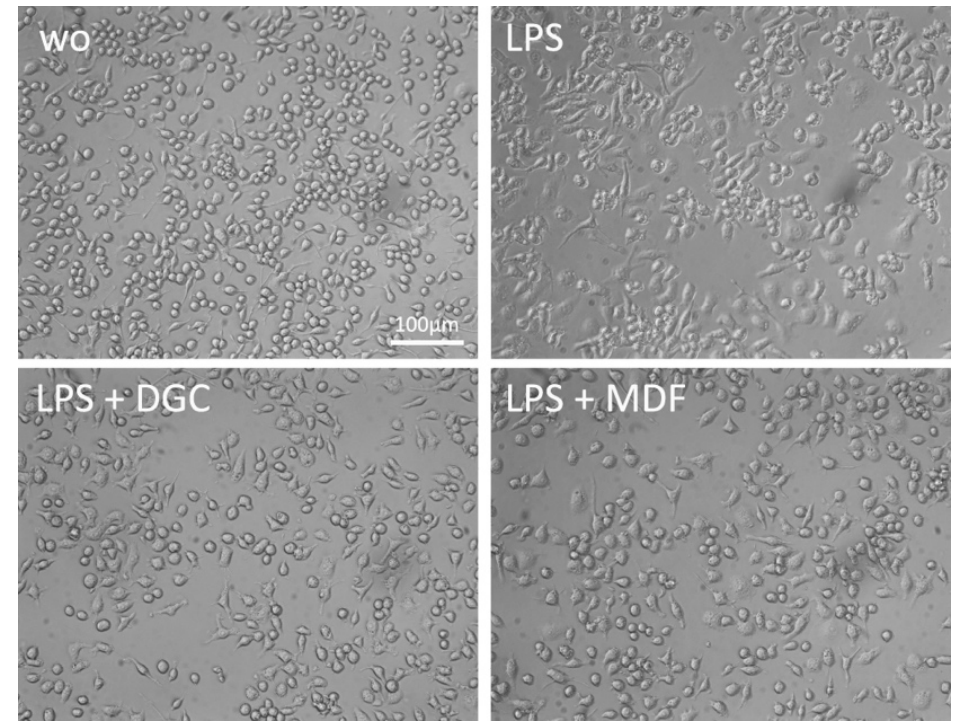

Figure 1. Morphological appearance of LPS-exposed RAW 264.7 macrophages in the presence of desalted DGC and MDF.

RAW 264.7 macrophages were exposed to $25 \%$ acid lysates of desalted DGC and MDF for $30 \mathrm{~min}$ followed by $100 \mathrm{ng} / \mathrm{mL}$ LPS for $24 \mathrm{~h}$. No detachment or any other obvious signs of cell death were noticed when LPS-exposed RAW 264.7 macrophages were grown in the presence of desalted DGC and MDF.

\subsection{Acid Lysates of DGC and MDF Reduce the Inflammatory Response of Macrophages}

Next, we investigated whether DGC and MDF can modulate the inflammatory response of macrophages. To this end, we exposed RAW 264.7 cells to the allograft lysates before the expression of pro-inflammatory cytokines was induced by LPS. DGC and MDF caused a reduction in the LPS-induced expression of IL1 and IL6, which was confirmed at the protein levels by IL6 immunoassay (Figure 2). Blocking of the TGF- $\beta$ receptor type I kinase with SB431542 failed to reverse the anti-inflammatory activity of the lysates (Supplementary Table S2). LPS initiated the nuclear translocation of p65 in RAW 264.7 cells, which was, however, only moderately reduced by DGC and MDF (Figure 3). Strong support for the anti-inflammatory activity of DGC and MDF comes from experiments with primary macrophages where DGC and MDF consistently reduced the expression of IL1 and IL6 of LPS-treated cells (Figure 4). 

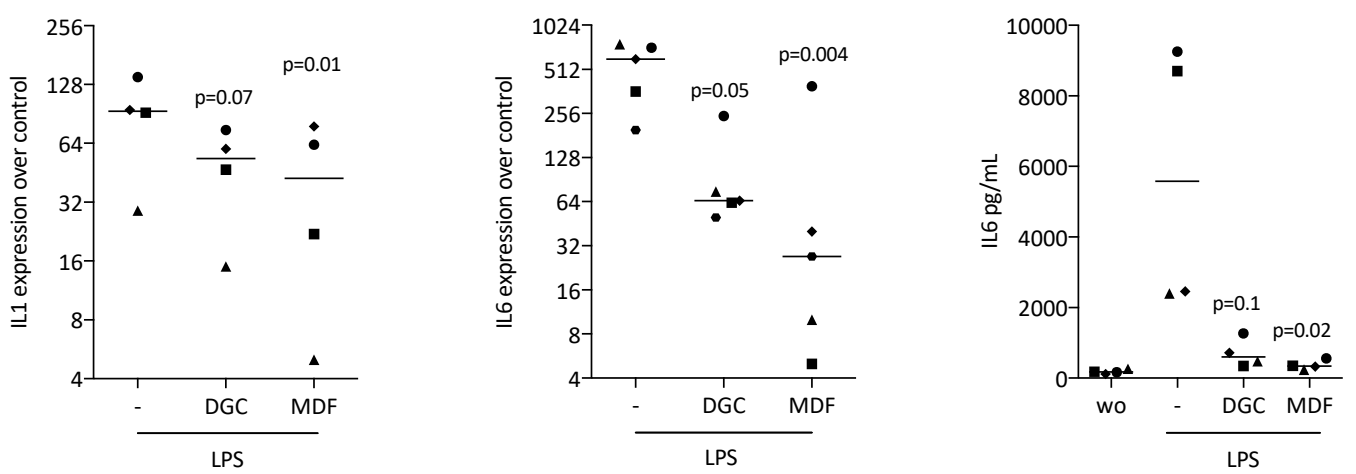

Figure 2. DGC and MDF reduced IL1 and IL6 in LPS-stimulated RAW 264.7 macrophages.

A

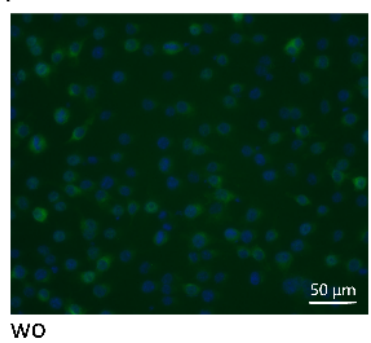

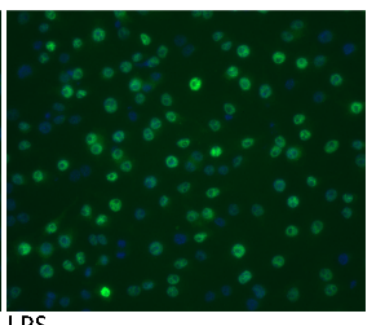

LPS


Figure 3. DGC and MDF weakly lowered p65 nuclear staining in LPS-stimulated RAW 264.7. (A) Immunostaining revealed the green fluorescence signals obtained with the p65 antibody. Nuclear staining with DAPI appears blue. (B) Mean signal intensity of nuclear staining by obtained by ImageJ software.
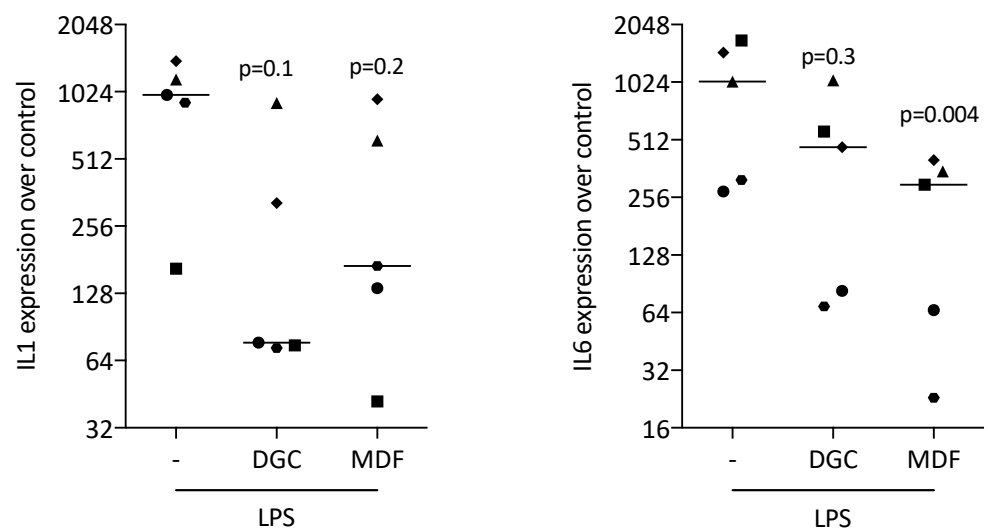

Figure 4. DGC and MDF reduced IL1 and IL6 in LPS-stimulated primary macrophages.

RAW 264.7 macrophages were exposed to $25 \%$ acid lysates of DGC and MDF for 30 min followed by $100 \mathrm{ng} / \mathrm{mL}$ LPS for $24 \mathrm{~h}$. Data show the relative expression changes normalized to the untreated cells. In addition, the IL6 ELISA data are shown. The experiments were performed four times, represented by a unique symbol for each replicate $(\mathrm{N}=4)$. The statistical analysis was based on a Friedmann test and $p$-values are indicated. The Wilcoxon matched-pairs signed rank test revealed $p>0.6$ when comparing DGC and MDF alone (not shown).

RAW 264.7 were exposed to LPS with or without DGC and MDF. WO means without and represents unstimulated cells.

The primary macrophages were exposed to 25\% acid lysates of DGC and MDF for $10 \mathrm{~min}$ followed by $100 \mathrm{ng} / \mathrm{mL}$ LPS for $24 \mathrm{~h}$. Data show the relative expression changes normalized to the untreated cells. The experiments were performed five times, represented by a unique symbol for each replicate $(\mathrm{N}=5)$. The statistical analysis was based on a 
Friedmann test and $p$-values are indicated. The Wilcoxon matched-pairs signed rank test revealed $p>0.6$ for IL1 and $p=0.063$ for IL6 when comparing DGC and MDF alone (not shown).

\subsection{Desalted Acid Lysates Reduce the Inflammatory Response of Macrophages}

Considering that the salt generated during the neutralization process may have suppressed the LPS-induced cytokine expression [27], we included a dose-response experiment with $\mathrm{NaCl}$ being added to the culture medium. Indeed, adding $100 \mathrm{mM}$ but not $30 \mathrm{mM}$ $\mathrm{NaCl}$ greatly diminished the LPS-induced expression of IL1 and IL6 by the RAW 264.7 cells (Table 1). Therefore, DGC and MDF containing $\mathrm{NaCl}$ produced by $\mathrm{pH}$ neutralization underwent desalting and buffer exchange before testing for a potential anti-inflammatory activity. As indicated in Figure 5, desalted DGC and MDF caused a reduction in the LPS-induced expression of IL1 and IL6. Thus, even though $\mathrm{NaCl}$ alone can reduce the expression of cytokines, the same was true for the desalted and buffer-exchanged preparations of DGC and MDF.

Table 1. Additional $\mathrm{NaCl}$ reduced the expression of IL1 and IL6 in RAW 264.7 macrophages.

\begin{tabular}{ccccc}
\hline $\begin{array}{c}\text { NaCl } \\
\text { Concentration }\end{array}$ & LPS + 0 mM & LPS + 10 $\mathbf{~ M M ~}$ & LPS + 30 mM & LPS + 100 mM \\
\hline $\begin{array}{c}\text { Experiment } 1 \\
\text { (IL1/IL6) } \\
\begin{array}{c}\text { Experiment 2 } \\
\text { (IL1/IL6) }\end{array}\end{array}$ & $170.7 / 422.1$ & $369.0 / 521.3$ & $807.2 / 594.4$ & $134.5 / 10.5$ \\
\hline
\end{tabular}
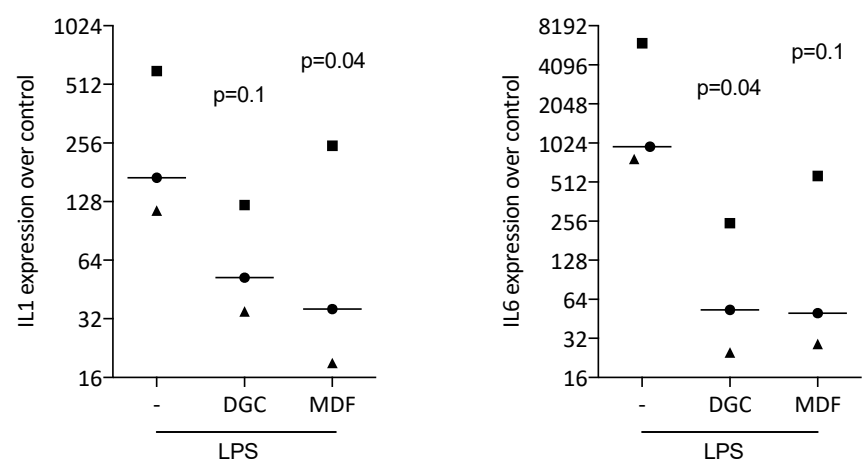

Figure 5. Desalted acid lysates of DGC and MDF reduced the inflammatory response of LPSstimulated RAW 264.7 macrophages.

RAW 264.7 macrophages were exposed to the indicated concentrations of $\mathrm{NaCl}$ being added to the culture medium for $30 \mathrm{~min}$ followed by $100 \mathrm{ng} / \mathrm{mL}$ LPS for $24 \mathrm{~h}$. Data show the relative expression changes normalized to the untreated cells. The experiments were performed two times, indicated as experiment 1 and 2.

RAW 264.7 macrophages were exposed to $25 \%$ concentrations of desalted and bufferexchanged acid lysates of DGC and MDF for $30 \mathrm{~min}$ followed by $100 \mathrm{ng} / \mathrm{mL}$ LPS for $24 \mathrm{~h}$. Data show the relative expression changes normalized to the untreated cells. The experiments were performed three times, represented by a unique symbol for each replicate. Statistical analysis was based on a Friedmann test and $p$-values are indicated. The Wilcoxon matched-pairs signed rank test revealed $p>0.99$ and $p=0.5$ for IL1 and IL6, respectively, when comparing DGC and MDF alone (not shown).

To rule out that the anti-inflammatory activity of the allografts was caused by the adsorption of LPS to the acid-soluble extracellular matrix, we pre-exposed the RAW 264.7 macrophages to LPS and then exposed them to the desalted DGC and MDF, and vice versa. In both settings, we saw an equivalent inhibition of IL1 $(72.8 \% \pm 4.7$ versus $67.5 \% \pm 12.1)$ and IL6 (96.2\% \pm 0.4 versus $95.9 \% \pm 1.1)$ expression by DGC. An equivalent 
inhibition of IL1 $(80.7 \% \pm 3.2$ versus $79.2 \% \pm 3.3)$ and IL6 $(94.3 \% \pm 2.3$ versus $95.5 \% \pm 0.6)$ expression was observed with MDF (data not shown).

\subsection{Acid Lysates of DGC and MDF Reduce the Inflammatory Response of ST2 Cells}

Finally, we tested whether or not DGC and MDF can dampen the inflammatory response of ST 2 cells and gingival fibroblasts. In ST 2 cells exposed to IL1 $\beta$ and TNF $\alpha$, DGC and MDF decreased the expression of IL6, iNOS and CCL5 (Figure 6). In gingival fibroblasts exposed to IL1 $\beta$ and TNF $\alpha$, DGC and MDF only moderately reduced the expression of IL6 and IL8 (Supplementary Figure S2). Moreover, DGC and MDF had no impact on the expression of the proliferation marker genes Ki67, PCNA and CCND1 in the gingival fibroblasts (data not shown). Thus, DGC and MDF can reduce the expression of IL6 and other inflammatory mediators in murine mesenchymal ST2 cells.
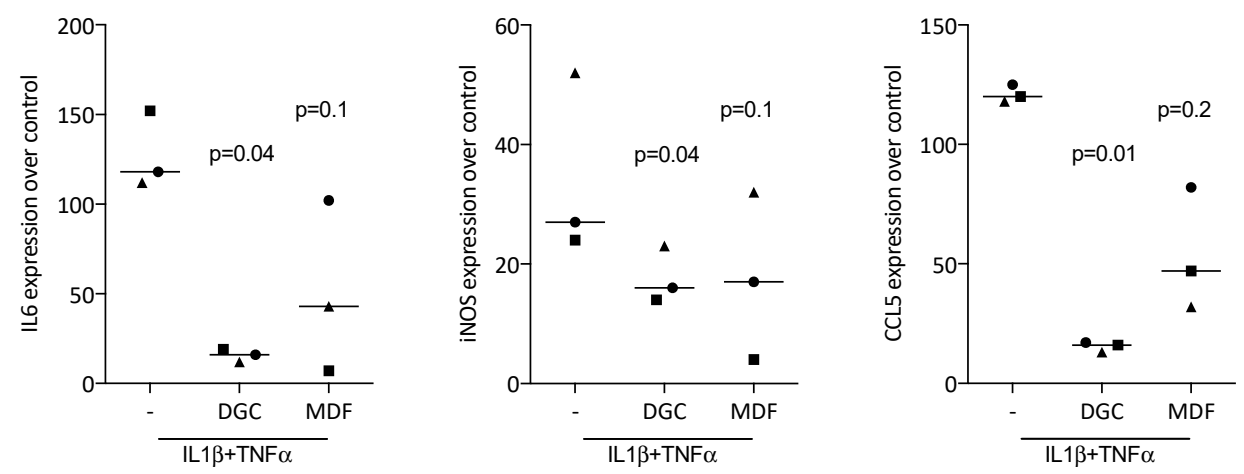

Figure 6. Desalted DGC and MDF reduced the expression of IL6, iNOS and CCL5 in ST2 cells.

ST2 cells were exposed to $25 \%$ desalted acid lysates of DGC and MDF for $30 \mathrm{~min}$ followed by $10 \mathrm{ng} / \mathrm{mL}$ of IL1 $\beta$ and TNF $\alpha$ for $24 \mathrm{~h}$. Data show the relative expression changes normalized to the untreated cells. The experiments were performed three times, represented by a unique symbol for each replicate $(\mathrm{N}=3)$. Statistical analysis was based on a Friedmann test and $p$-values are indicated. The Wilcoxon matched-pairs signed rank test revealed $p=0.5, p=0.2$ and $p>0.9$ for IL6, iNOS and CCL5, respectively, when comparing DGC and MDF alone (not shown).

\section{Discussion}

Demineralized bone matrix [1] may have biological activities that remain overshadowed by the impressive osteoinductive properties when transplanted ectopically in a rodent model $[9,10]$. In a clinical scenario, however, demineralized freeze-dried bone allografts failed to exert the hallmark signs of osteoinductivity [28]. Moreover, demineralized teeth are proposed as grafts for bone reconstruction $[17,29]$. DBM research is closely related to the discovery of the BMPs $[1,7,30]$, yet attempts to identify other growth factors that survive the demineralization process are minor $[13,31,32]$. Surprisingly, however, even though the early stages of graft consolidation occur in a catabolic inflammatory environment [15], we lack research on whether DBM might influence the process of inflammation. We therefore tested the potential anti-inflammatory properties of acid lysates prepared from DGC and MDF based on our established bioassays.

The main finding of the present research was that acid lysates from granules and fibers reduced the inflammatory response of RAW 264.7 macrophages, particularly IL1 and IL6 expression. This anti-inflammatory activity was not restricted to RAW 264.7 macrophages as the LPS-induced expression of inflammatory cytokines in the primary macrophages was also diminished by DGC and MDF. Care should be taken when interpreting these findings because $\mathrm{NaCl}$ generated by the neutralization of $\mathrm{HCl}$ might have anti-inflammatory properties; however, it needs around $200 \mathrm{mM} \mathrm{NaCl}$ to cause an M1 to M2 shift of primary human macrophages [27]. We confirmed that adding $300 \mathrm{mM} \mathrm{NaCl}$ to the culture medium is toxic to RAW 264.7 macrophages and observed an anti-inflammatory activity by adding $100 \mathrm{mM}$ 
$\mathrm{NaCl}$ to the culture medium. To rule out that the anti-inflammatory activity of DGC and MDF is an artefact caused by the disbalanced salt concentration of the culture medium, the lysates were subjected to desalting and buffer exchange for serum-free medium. In support of the overall conclusion, desalted and buffer-exchanged DGC and MDF greatly reduced the LPS-induced expression of IL1 and IL6 in RAW 264.7 macrophages.

To rule out that DGC and MDF bind to LPS and thereby neutralize its activity, we first exposed the RAW 264.7 macrophages to LPS before adding the lysates. In this setting, the desalted lysates of both allografts had an anti-inflammatory activity with respect to the expression of IL1 and IL6 in the RAW 264.7 macrophages. To further rule out this possibility, we implemented the ST2 murine mesenchymal cell line where IL1 $\beta$ and TNF $\alpha$ can provoke an inflammatory response [19]. We show here that with ST2 cells, desalted DGC and MDF significantly attenuated the expression of IL6, iNOS and CCL5. Together, these findings suggest that the anti-inflammatory activity of the allograft lysates cannot be simply blamed on the neutralization of LPS. Moreover, these data extend our observation obtained with macrophages towards mesenchymal cells. Surprisingly, however, the inflammatory response of human gingival fibroblast to IL1 $\beta$ and TNF $\alpha$ was only moderately reduced by DGC and MDF. These findings suggest that the murine cells are potentially more sensitive than human cells to lysates of DGC and MDF in their activity in terms of reducing the expression of inflammatory mediators.

The present study has limitations. Even though we describe here an anti-inflammatory effect of acid lysates of DGC and MDF in macrophages and ST2 cells, many questions remain to be answered. First, we have not yet identified a specific molecule in DGC and MDF that is responsible for the anti-inflammatory activity. TGF- $\beta$ signaling can be ruled out because blocking of the TGF- $\beta$ receptor type I kinase with SB431542 failed to reverse the anti-inflammatory activity. Future studies should, therefore, include a size fractionation of the acid lysate to identify the molecular weight of the target molecule, followed by a proteomic analysis to identify possible candidates. However, this approach requires a high sample volume, which was not available when conducting the present study. Second, and consequently, the signaling cascade that leads to the reduction in the inflammatory response needs further refinement, and our data, which show only a moderate blocking of LPS-induced p65 nuclear translocation in RAW 264.7 cells, are not convincing. Third, we did not include allografts from other manufacturers, which is a limitation because each provider has slightly different protocols to prepare demineralized bone. Fourth, the reason why murine macrophages and ST2 cells, but not human gingival fibroblasts show this response remains enigmatic. Finally, we cannot tell if the anti-inflammatory activity we observed in vitro translates to in vivo, and if yes, the overall biological and clinical consequences need to be discovered in future studies. The present research should, therefore, be considered a preliminary screening approach showing that acid lysates of allografts can dampen the simulated inflammatory response of murine macrophages and mesenchymal cells under in vitro conditions.

Supplementary Materials: The following supporting information can be downloaded at: https: / / www.mdpi.com/article/10.3390/app12020876/s1, Figure S1: DGC and MDF maintain the viability of RAW 264.7 macrophages; Figure S2: DGC and MDF moderately reduce the expression of IL6 and IL8 in IL1 $\beta$ and TNF $\alpha$-stimulated gingival fibroblasts; Table S1: Primer sequences of proliferation marker genes; Table S2: Blocking of the TGF- $\beta$ receptor type I kinase with SB431542 failed to reverse the anti-inflammatory activity of the DGC lysate.

Author Contributions: Conceptualization, L.P. and R.G.; data curation, L.P.; formal analysis, L.P. and R.G.; methodology, L.P., A.O., A.O.A. and Z.K.; project administration, L.P., A.O., A.O.A. and J.N.; supervision, L.P. and R.G.; validation, L.P., J.N. and Z.K.; visualization, L.P.; writing-original draft, L.P. and R.G.; writing-review and editing, L.P., A.O., A.O.A., J.N., Z.K. and R.G. All authors have read and agreed to the published version of the manuscript.

Funding: The research was funded by the Medical University of Vienna, University Clinic of Dentistry, Vienna, Austria. 
Institutional Review Board Statement: The study was conducted according to the guidelines of the Declaration of Helsinki, and approved by the Ethics Committee of the Medical University of Vienna (EK NR 631/2007).

Informed Consent Statement: Informed consent was obtained from all subjects involved in the study.

Data Availability Statement: All data are available on demand.

Acknowledgments: This research was supported by LifeNet Health Europe GmbH, Vienna, Austria by providing the biomaterial.

Conflicts of Interest: The authors declare no conflict of interest.

\section{References}

1. Gruskin, E.; Doll, B.A.; Futrell, F.W.; Schmitz, J.P.; Hollinger, J.O. Demineralized bone matrix in bone repair: History and use. Adv. Drug Deliv. Rev. 2012, 64, 1063-1077. [CrossRef]

2. Cockerham, B.L., 3rd; Patel, A.; Greenwell, H.; Hill, M.; Shumway, B.; Hsu, H.T. Ridge Augmentation Comparing a Cancellous Block Allograft to an Osteoinductive Demineralized Bone Matrix Allograft: A Randomized, Controlled, Blinded Clinical Trial. Int. J. Periodontics Restor. Dent. 2020, 40, 571-578. [CrossRef]

3. Patel, A.; Greenwell, H.; Hill, M.; Shumway, B.; Radmall, A. Ridge Augmentation Comparing an Allograft Plus Autogenous Bone Chips to an Osteoinductive Demineralized Bone Matrix: A Clinical and Histologic Study in Humans. Implant. Dent. 2019, 28, 613-620. [CrossRef]

4. Brink, O. The choice between allograft or demineralized bone matrix is not unambiguous in trauma surgery. Injury 2021, 52 (Suppl. 2), S23-S28. [CrossRef]

5. Shepard, N.A.; Rush, A.J., 3rd; Scarborough, N.L.; Carter, A.J.; Phillips, F.M. Demineralized Bone Matrix in Spine Surgery: A Review of Current Applications and Future Trends. Int. J. Spine Surg. 2021, 15, 113-119. [CrossRef]

6. Buser, D.; Hoffmann, B.; Bernard, J.P.; Lussi, A.; Mettler, D.; Schenk, R.K. Evaluation of filling materials in membrane-protected bone defects. A comparative histomorphometric study in the mandible of miniature pigs. Clin. Oral Implant. Res. 1998, 9, 137-150. [CrossRef] [PubMed]

7. Urist, M.R. Bone: Formation by autoinduction. Science 1965, 150, 893-899. [CrossRef] [PubMed]

8. Sampath, T.K.; Reddi, A.H. Discovery of bone morphogenetic proteins-A historical perspective. Bone 2020, 140, 115548. [CrossRef] [PubMed]

9. Schouten, C.C.; Hartman, E.H.; Spauwen, P.H.; Jansen, J.A. DBM induced ectopic bone formation in the rat: The importance of surface area. J. Mater. Sci. Mater. Med. 2005, 16, 149-152. [CrossRef]

10. Nishimoto, S.K.; Chang, C.H.; Gendler, E.; Stryker, W.F.; Nimni, M.E. The effect of aging on bone formation in rats: Biochemical and histological evidence for decreased bone formation capacity. Calcif. Tissue Int. 1985, 37, 617-624. [CrossRef] [PubMed]

11. Pfeilschifter, J.; Diel, I.; Scheppach, B.; Bretz, A.; Krempien, R.; Erdmann, J.; Schmid, G.; Reske, N.; Bismar, H.; Seck, T.; et al. Concentration of transforming growth factor beta in human bone tissue: Relationship to age, menopause, bone turnover, and bone volume. J. Bone Miner. Res. 1998, 13, 716-730. [CrossRef] [PubMed]

12. Strauss, F.J.; Stahli, A.; Beer, L.; Mitulovic, G.; Gilmozzi, V.; Haspel, N.; Schwab, G.; Gruber, R. Acid bone lysate activates TGFbeta signalling in human oral fibroblasts. Sci. Rep. 2018, 8, 16065. [CrossRef] [PubMed]

13. Panahipour, L.; Omerbasic, A.; Nasirzade, J.; Gruber, R. TGF-beta Activity of a Demineralized Bone Matrix. Int. J. Mol. Sci. 2021, 22, 664. [CrossRef]

14. Khera, T.K.; Dick, A.D.; Nicholson, L.B. Fragile X-related protein FXR1 controls post-transcriptional suppression of lipopolysaccharide-induced tumour necrosis factor-alpha production by transforming growth factor-beta1. FEBS J. 2010, 277, 2754-2765. [CrossRef] [PubMed]

15. Einhorn, T.A.; Gerstenfeld, L.C. Fracture healing: Mechanisms and interventions. Nat. Rev. Rheumatol. 2015, 11, 45-54. [CrossRef] [PubMed]

16. Gruber, R. Osteoimmunology: Inflammatory osteolysis and regeneration of the alveolar bone. J. Clin. Periodontol. 2019, 46 (Suppl. 21), 52-69. [CrossRef]

17. Nasirzade, J.; Kargarpour, Z.; Mitulovic, G.; Strauss, F.J.; Panahipour, L.; Schwarz, F.; Gruber, R. Proteomic and genomic analysis of acid dentin lysate with focus on TGF-beta signaling. Sci. Rep. 2021, 11, 12247. [CrossRef] [PubMed]

18. Panahipour, L.; Kochergina, E.; Kreissl, A.; Haiden, N.; Gruber, R. Milk modulates macrophage polarization in vitro. Cytokine X 2019, 1, 100009. [CrossRef]

19. Kargarpour, Z.; Nasirzade, J.; Panahipour, L.; Miron, R.J.; Gruber, R. Platelet-Rich Fibrin Decreases the Inflammatory Response of Mesenchymal Cells. Int. J. Mol. Sci. 2021, 22, 11333. [CrossRef]

20. Panahipour, L.; Nasserzare, S.; Amer, Z.; Brucke, F.; Stahli, A.; Kreissl, A.; Haiden, N.; Gruber, R. The anti-inflammatory effect of milk and dairy products on periodontal cells: An in vitro approach. Clin. Oral. Investig. 2019, 23, 1959-1966. [CrossRef]

21. Kargarpour, Z.; Nasirzade, J.; Panahipour, L.; Miron, R.J.; Gruber, R. Liquid PRF Reduces the Inflammatory Response and Osteoclastogenesis in Murine Macrophages. Front. Immunol. 2021, 12, 636427. [CrossRef] [PubMed] 
22. Zhao, R.; Liang, H.; Clarke, E.; Jackson, C.; Xue, M. Inflammation in Chronic Wounds. Int. J. Mol. Sci. 2016, 17, 2085. [CrossRef]

23. Schett, G.; Neurath, M.F. Resolution of chronic inflammatory disease: Universal and tissue-specific concepts. Nat. Commun. 2018, 9, 3261. [CrossRef] [PubMed]

24. Baldik, Y.; Diwan, A.D.; Appleyard, R.C.; Fang, Z.M.; Wang, Y.; Murrell, G.A. Deletion of iNOS gene impairs mouse fracture healing. Bone 2005, 37, 32-36. [CrossRef]

25. Kimura, K.; Nagano, M.; Salazar, G.; Yamashita, T.; Tsuboi, I.; Mishima, H.; Matsushita, S.; Sato, F.; Yamagata, K.; Ohneda, O. The role of CCL5 in the ability of adipose tissue-derived mesenchymal stem cells to support repair of ischemic regions. Stem. Cells Dev. 2014, 23, 488-501. [CrossRef] [PubMed]

26. Saxena, R.K.; Vallyathan, V.; Lewis, D.M. Evidence for lipopolysaccharide-induced differentiation of RAW264.7 murine macrophage cell line into dendritic like cells. J. Biosci. 2003, 28, 129-134. [CrossRef]

27. Amara, S.; Whalen, M.; Tiriveedhi, V. High salt induces anti-inflammatory MPhi2-like phenotype in peripheral macrophages. Biochem. Biophys. Rep. 2016, 7, 1-9. [CrossRef]

28. Piattelli, A.; Scarano, A.; Corigliano, M.; Piattelli, M. Comparison of bone regeneration with the use of mineralized and demineralized freeze-dried bone allografts: A histological and histochemical study in man. Biomaterials 1996, 17, 1127-1131. [CrossRef]

29. Hazballa, D.; Inchingolo, A.D.; Inchingolo, A.M.; Malcangi, G.; Santacroce, L.; Minetti, E.; Di Venere, D.; Limongelli, L.; Bordea, I.R.; Scarano, A.; et al. The effectiveness of autologous demineralized tooth graft for the bone ridge preservation: A systematic review of the literature. J. Biol. Regul. Homeost. Agents. 2021, 35, 283-294. [CrossRef]

30. Wozney, J.M.; Rosen, V.; Celeste, A.J.; Mitsock, L.M.; Whitters, M.J.; Kriz, R.W.; Hewick, R.M.; Wang, E.A. Novel regulators of bone formation: Molecular clones and activities. Science 1988, 242, 1528-1534. [CrossRef]

31. Wildemann, B.; Kadow-Romacker, A.; Haas, N.P.; Schmidmaier, G. Quantification of various growth factors in different demineralized bone matrix preparations. J. Biomed. Mater. Res. A 2007, 81, 437-442. [CrossRef] [PubMed]

32. Wildemann, B.; Kadow-Romacker, A.; Pruss, A.; Haas, N.P.; Schmidmaier, G. Quantification of growth factors in allogenic bone grafts extracted with three different methods. Cell Tissue Bank. 2007, 8, 107-114. [CrossRef] [PubMed] 